\title{
Racial/ethnic differences in multiple-gene sequencing results for hereditary cancer risk
}

\author{
Jennifer L. Caswell-Jin, MD¹, Tanya Gupta, MD ${ }^{1}$, Evan Hall, MD ${ }^{1}$, Iva M. Petrovchich, BS ${ }^{1}$, \\ Meredith A. Mills, BA ${ }^{1}$, Kerry E. Kingham, MS ${ }^{1}$, Rachel Koff, MS ${ }^{1}$, Nicolette M. Chun, MS ${ }^{1}$, \\ Peter Levonian, $\mathrm{MS}^{1}$, Alexandra P. Lebensohn, $\mathrm{MS}^{1}$, James $\mathrm{M}$. Ford, $\mathrm{MD}^{1}$ and \\ Allison W. Kurian, MD, MSc ${ }^{1}$
}

\begin{abstract}
Purpose: We examined racial/ethnic differences in the usage and results of germ-line multiple-gene sequencing (MGS) panels to evaluate hereditary cancer risk.

Methods: We collected genetic testing results and clinical information from 1,483 patients who underwent MGS at Stanford University between 1 January 2013 and 31 December 2015.

Results: Asians and Hispanics presented for MGS at younger ages than whites (48 and 47 vs. $55 ; P=5 \mathrm{E}-16$ and 5E-14). Across all panels, the rate of pathogenic variants (15\%) did not differ significantly between racial groups. Rates by gene did differ: in particular, a higher percentage of whites than nonwhites carried pathogenic $C H E K 2$ variants $(3.8 \%$ vs. $1.0 \%$; $P=0.002)$. The rate of a variant of uncertain significance (VUS) result was higher in nonwhites than whites ( $36 \%$ vs. $27 \%$; $P=2 \mathrm{E}-4)$. The probability of
\end{abstract}

a VUS increased with increasing number of genes tested; this effect was more pronounced for nonwhites than for whites $(1.1 \%$ absolute difference in VUS rates testing BRCA1/2 vs. $8 \%$ testing 13 genes vs. $14 \%$ testing 28 genes), worsening the disparity.

Conclusion: In this diverse cohort undergoing MGS testing, pathogenic variant rates were similar between racial/ethnic groups. By contrast, VUS results were more frequent among nonwhites, with potential significance for the impact of MGS testing by race/ ethnicity.

Genet Med advance online publication 27 July 2017

Key Words: hereditary cancer panel; multiple-gene panel testing; next-generation sequencing; race/ethnicity; variants of unknown significance

\section{INTRODUCTION}

With the recent development of next-generation sequencing technology, multiple commercial laboratories have begun to offer multiple-gene sequencing (MGS) panels for hereditary cancer risk assessment. Rapidly, the paradigm in cancer genetics clinics shifted from testing selected patients for specific high-risk cancer syndromes (including hereditary breast and ovarian cancer and Lynch, Li-Fraumeni, and Cowden syndromes) to testing most patients simultaneously for multiple high-risk cancer syndromes as well as moderaterisk cancer-associated genes. Recent studies have demonstrated that MGS panels regularly discover unsuspected highor moderate-risk pathogenic variants for which patients had no clinical testing indication. ${ }^{1-12}$ In addition to the potential clinical utility of an incidental high-risk result, moderate-risk pathogenic variant results increasingly guide cancer screening recommendations. ${ }^{13,14}$ However, the information gain from MGS must be weighed against its cost of greater uncertainty, with 10 times more variants of uncertain significance (VUS) than arise when testing BRCA1 and BRCA2 (BRCA1/2) only. ${ }^{4-6,8,9,11,12}$

In larger studies $(N>1,000)$ of hereditary cancer panel testing, the study populations were predominantly nonHispanic white. ${ }^{2,3,7,9,11}$ While some studies have examined ethnically diverse populations, ${ }^{1,8}$ to our knowledge no study has extensively characterized differences in MGS results by race/ethnicity. Yet it is increasingly clear that racial/ethnic disparities in genomic sequencing access perpetuate inaccurate or misleading sequencing results for racial/ethnic minority patients. ${ }^{15,16}$ We took advantage of a large, diverse, contemporary cohort of patients who underwent clinical MGS from 2013 to 2015 to examine differences in clinical presentation and genetic testing results by race/ethnicity.

\section{MATERIALS AND METHODS}

\section{Patients}

Study participants were consecutive patients who had genetic testing at the Stanford University Clinical Cancer Genetics Program from 1 January 2013 to 31 December 2015 $(N=2,239)$. Patients provided informed consent under study protocols approved by the Stanford University Institutional Review Board. The majority of patients presented to the clinic because they met guidelines of the National Comprehensive Cancer Network (NCCN) for cancer genetics referral, ${ }^{17,18}$ while a minority presented because of interest in a clinical trial available at our institution that allowed MGS testing not otherwise covered by insurance (ClinicalTrials.gov NCT02324062). ${ }^{19}$ Genetic testing was then sent if a patient

${ }^{1}$ Department of Medicine, Division of Medical Oncology, Stanford University, Stanford, California, USA. Correspondence: Allison W. Kurian (akurian@stanford.edu) 
met criteria per NCCN guidelines for testing of a genetic syndrome such as hereditary breast and ovarian syndrome or Lynch syndrome, ${ }^{17,18}$ if they enrolled on the clinical trial, or per patient interest with self-pay. The majority of these patients $(N=1,483)$ underwent hereditary cancer genetic panel testing and were included in further analyses. We defined a panel as containing at least six genes, including at least one that was not part of a named hereditary cancer syndrome: hereditary breast and ovarian syndrome (BRCA1/2), Lynch syndrome ( $M L H 1 /$ MSH2/MSH6/PMS2/EPCAM), familial adenomatous polyposis (APC/MYH), Li-Fraumeni syndrome (TP53), hereditary diffuse gastric cancer $(C D H 1)$, familial atypical multiple mole melanoma syndrome (CDKN2A), Cowden syndrome (PTEN), and Peutz-Jeghers syndrome (STK11). Of those patients from the initial cohort excluded for undergoing genetic testing other than panel testing, the majority underwent testing either for BRCA1/2 alone $(N=326)$ or for a single gene, generally their family's known mutation $(N=307)$ (Supplementary Figure $\mathbf{S} 1$ online).

Information on demographics-including race/ethnicity by self-report-, personal and family cancer history, and tumor characteristics were recorded at the clinic visit. We classified as "white" those patients who reported their race as white and their ethnicity as neither Hispanic nor Ashkenazi Jewish (i.e., non-Hispanic, non-Ashkenazi white) $(N=751)$. For purposes of this analysis, we considered patients of self-reported Ashkenazi Jewish descent separately from other whites, given their distinct genetic background. For all analyses of nonwhite patients as a group, we included all individuals whose race/ ethnicity was known and was not non-Hispanic, nonAshkenazi white: this included Asians $(N=288)$, Hispanics $(N=213)$, Ashkenazi Jews $(N=149)$, Native Americans $(N=33)$, African Americans $(N=25)$, Hawaiian/Pacific Islanders $(N=10)$, and individuals who listed their race as "other" $(N=8)$.

\section{Genetic panel testing}

Gene sequencing and variant classification were performed by one of several commercially available laboratories, as determined by the ordering clinician, with genetic results linked to patient information in a secure, anonymized research database. For each panel ordered, if at least one gene had a pathogenic or likely pathogenic variant, the result of the entire panel was considered pathogenic; if no gene was reported as having a pathogenic or likely pathogenic variant and at least one gene was reported as having a VUS, the result of the panel was considered VUS; and if all genes were reported as having no variants or benign variants only, the result of the panel was considered benign.

\section{Statistical analysis}

All statistical analyses were performed with the $\mathrm{R}$ programming language V3.2.3. Differences between races/ethnicities were compared using two-sided $t$ tests (continuous variables) and $\chi^{2}$ tests (categorical variables). Within each set of comparisons, $P$ values were adjusted for multiple hypothesis testing using the Benjamini-Hochberg procedure (false discovery rate). Unadjusted $P$ values were reported, but only those with false discovery rate $<0.05$ were described as significant, while those that were $<0.05$ but with false discovery rate of $0.05-0.1$ were described as trends.

\section{RESULTS}

\section{Demographics}

Nearly half the cohort (49\%) was nonwhite, including 288 Asians (19\%), 213 Hispanics (14\%), 149 Ashkenazi Jews (10\%), 33 Native Americans (2\%), and 25 African Americans (2\%). The cohort was predominantly female (92\%), with a majority (60\%) having a personal history of breast cancer. Demographic details of the cohort by race/ethnicity are shown in Table 1.

We assessed for racial/ethnic differences in the characteristics of the panel-tested population, comparing each nonwhite ethnic group of at least 100 patients (Asians, Hispanics, and Ashkenazi Jews) to the white population for differences in gender, age at testing, personal history of cancer, family history of any cancer, and family history of breast cancer (15 total tests). White and Ashkenazi Jewish patients were tested at significantly older ages (mean age 55) than Asians (48) $(P=5 \mathrm{E}-16)$ and Hispanics $(47)(P=5 \mathrm{E}-14)$. The age difference was present both in patients with a personal history of breast cancer (mean age 56 for whites vs. 49 for Asians and 50 for Hispanics; $P=2 \mathrm{E}-10$ and $2 \mathrm{E}-6)$ and in those without (mean age 54 for whites vs. 48 for Asians and 43 for Hispanics; $P=8 \mathrm{E}-6$ and $1 \mathrm{E}-9$ ). A higher proportion of the Ashkenazi Jewish patients than other white patients who underwent panel testing had no personal history of cancer (36\% vs. $20 \%$; $P=4 \mathrm{E}-5$ ). Finally, Asians more often reported no family history of cancer than did whites ( $9 \%$ vs. $4 \%$; $P=7 \mathrm{E}-4$ ), and similarly, more often reported no family history of breast cancer than did whites (55\% vs. $69 \%$, $\mathrm{P}=3 \mathrm{E}-5)$. As expected, patients who reported a family history of breast cancer were on average modestly older than those who did not (53 vs. 51, $P=4 \mathrm{E}-4$ ); however, in a multivariate analysis including race (Asian versus white) and age at testing as predictors of the presence or absence of a family history of breast cancer, race $(P=2 \mathrm{E}-4)$ was a stronger predictor than age $(P=0.04)$, suggesting that the difference in family history between Asians and whites reflected more than a difference in age at testing.

\section{Genetic panel testing results by race/ethnicity}

Most patients underwent cross-cancer panel (55\%) or breast/ gynecologic cancer panel (41\%) testing; a complete description of panels is provided in Supplementary Table S1. Panel size ranged from 6 to 62 genes, with a median of 25 (Supplementary Figure S2). Asian patients had slightly fewer genes sent on their panels (mean 23.2 genes vs. 24.9 genes for white patients; $P=0.002$ ) (mean number of genes on a panel was 24.4 for Hispanics and 25.1 for Ashkenazi Jews.)

Across all panels, the frequency of a pathogenic variant was $15 \%$, and this was similar across different races/ethnicities (whites: 14\%, Asians: 13\%, Hispanics: 21\%, Ashkenazi Jews: 
15\%) (Figure 1a). The trend toward a higher percentage of Hispanics receiving pathogenic variants $(P=0.02$ versus whites) resulted mainly from the high rate of pathogenic
BRCA1 variants in Hispanics $(6.3 \%$ vs. $1.9 \%$ in whites; Supplementary Table S2). Across all panels, the VUS rate was $31 \%$, and was higher for nonwhites (36\%) than whites

Table 1 Racial/ethnic differences in demographics of 1,483 patients undergoing multigene panel testing

\begin{tabular}{|c|c|c|c|c|c|c|}
\hline & Total cohort & Non-Hispanic white & Asian & Hispanic & Ashkenazi Jewish & Other \\
\hline & 1,483 & $751(51 \%)$ & $288(19 \%)$ & $213(14 \%)$ & $149(10 \%)$ & $76(5 \%)$ \\
\hline \multicolumn{7}{|l|}{ Gender } \\
\hline Female & 1,366 (92\%) & $691(92 \%)$ & $266(92 \%)$ & $197(92 \%)$ & $135(91 \%)$ & 72 (95\%) \\
\hline \multicolumn{7}{|l|}{ Age at testing (years) } \\
\hline$<30$ & $48(3 \%)$ & $16(2 \%)$ & $9(3 \%)$ & $13(6 \%)$ & $7(5 \%)$ & $3(4 \%)$ \\
\hline $50-59$ & $422(28 \%)$ & $231(31 \%)$ & $68(24 \%)$ & $49(23 \%)$ & $45(30 \%)$ & $27(36 \%)$ \\
\hline$>59$ & $420(28 \%)$ & $274(36 \%)$ & $44(15 \%)$ & $36(17 \%)$ & $52(35 \%)$ & $14(18 \%)$ \\
\hline \multicolumn{7}{|c|}{ Personal history of cancer } \\
\hline None & $317(21 \%)$ & $153(20 \%)$ & $42(15 \%)$ & $48(23 \%)$ & $54(36 \%)$ & $20(26 \%)$ \\
\hline Breast only & $772(52 \%)$ & $388(52 \%)$ & $174(60 \%)$ & $103(48 \%)$ & $59(40 \%)$ & $46(61 \%)$ \\
\hline Multiple cancer types & $175(12 \%)$ & $97(13 \%)$ & $28(10 \%)$ & $28(13 \%)$ & $17(11 \%)$ & $4(5 \%)$ \\
\hline \multicolumn{7}{|l|}{ Family history of cancer } \\
\hline None & $73(5 \%)$ & $27(4 \%)$ & $26(9 \%)$ & $17(8 \%)$ & $0(0 \%)$ & $3(4 \%)$ \\
\hline Breast only & $101(7 \%)$ & $45(6 \%)$ & $29(10 \%)$ & $9(4 \%)$ & $13(9 \%)$ & $5(7 \%)$ \\
\hline Breast and other & $878(59 \%)$ & $473(63 \%)$ & $129(45 \%)$ & $126(59 \%)$ & $94(63 \%)$ & $51(67 \%)$ \\
\hline Non-breast cancers & $431(29 \%)$ & $206(27 \%)$ & $104(36 \%)$ & $61(29 \%)$ & $42(28 \%)$ & $17(22 \%)$ \\
\hline
\end{tabular}

The "other" category includes 33 Native Americans, 25 African Americans, 10 Hawaiian or Pacific Islanders, and 8 individuals who listed their race or ethnicity as "other." Six patients with unknown race or ethnicity were included in the total cohort but excluded from the subsequent race/ethnicity categories.

a
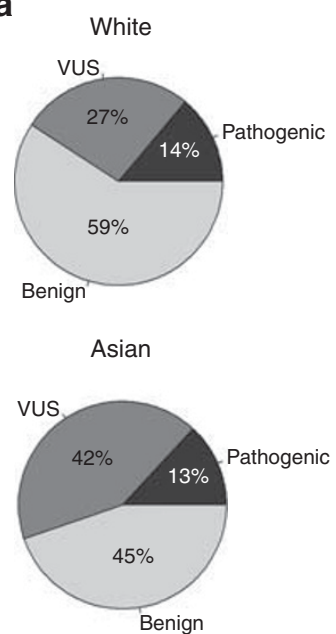

Ashkenazi ancestry
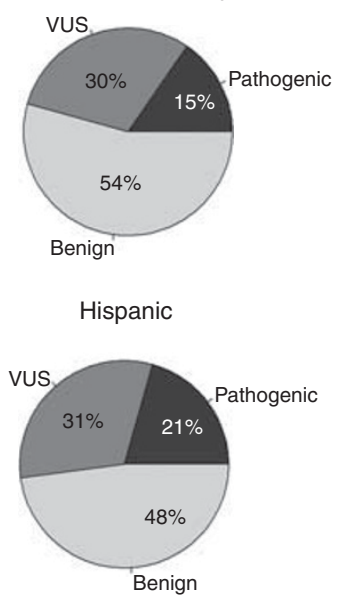

b

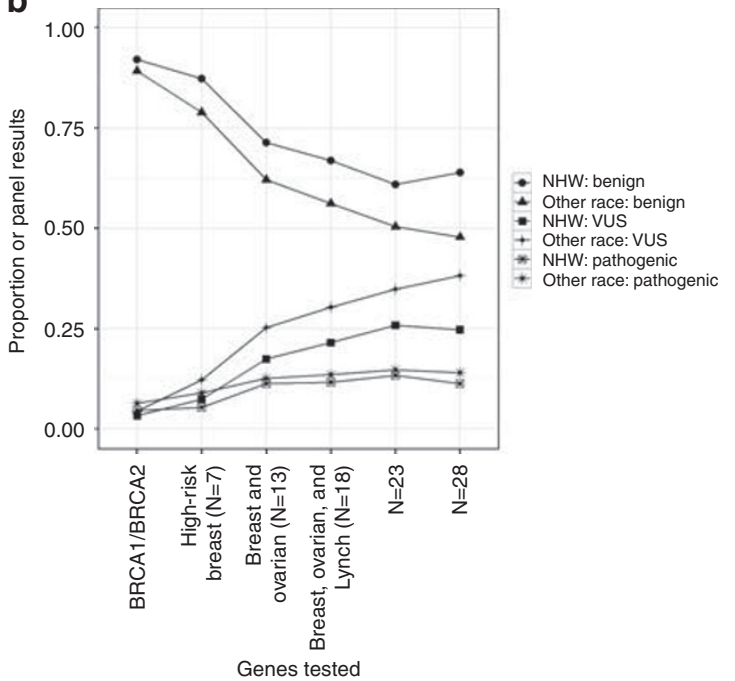

Figure 1 Panel testing results by race/ethnicity. (a) Results across all panels. Percentages may not add up to 100\% because of rounding error. (b) Change in panel testing results with increasing number of genes tested. "High-risk breast" includes TP53, CDH1, PTEN, STK11, and PALB2 (in addition to BRCA1/2); "Breast and ovarian" adds ATM, CHEK2, NBN, RAD51C, RAD51D, and BRIP1; "Breast, ovarian, and Lynch" adds MLH1, MSH2, MSH6, PMS2, and EPCAM; $N=23$ adds five randomly selected genes; $N=28$ adds five more randomly selected genes. "Other race" includes white patients of Hispanic or Ashkenazi Jewish descent, as well as patients of Asian, African, and Native American descent. NHW, non-Hispanic white; VUS, variant of unknown significance. 


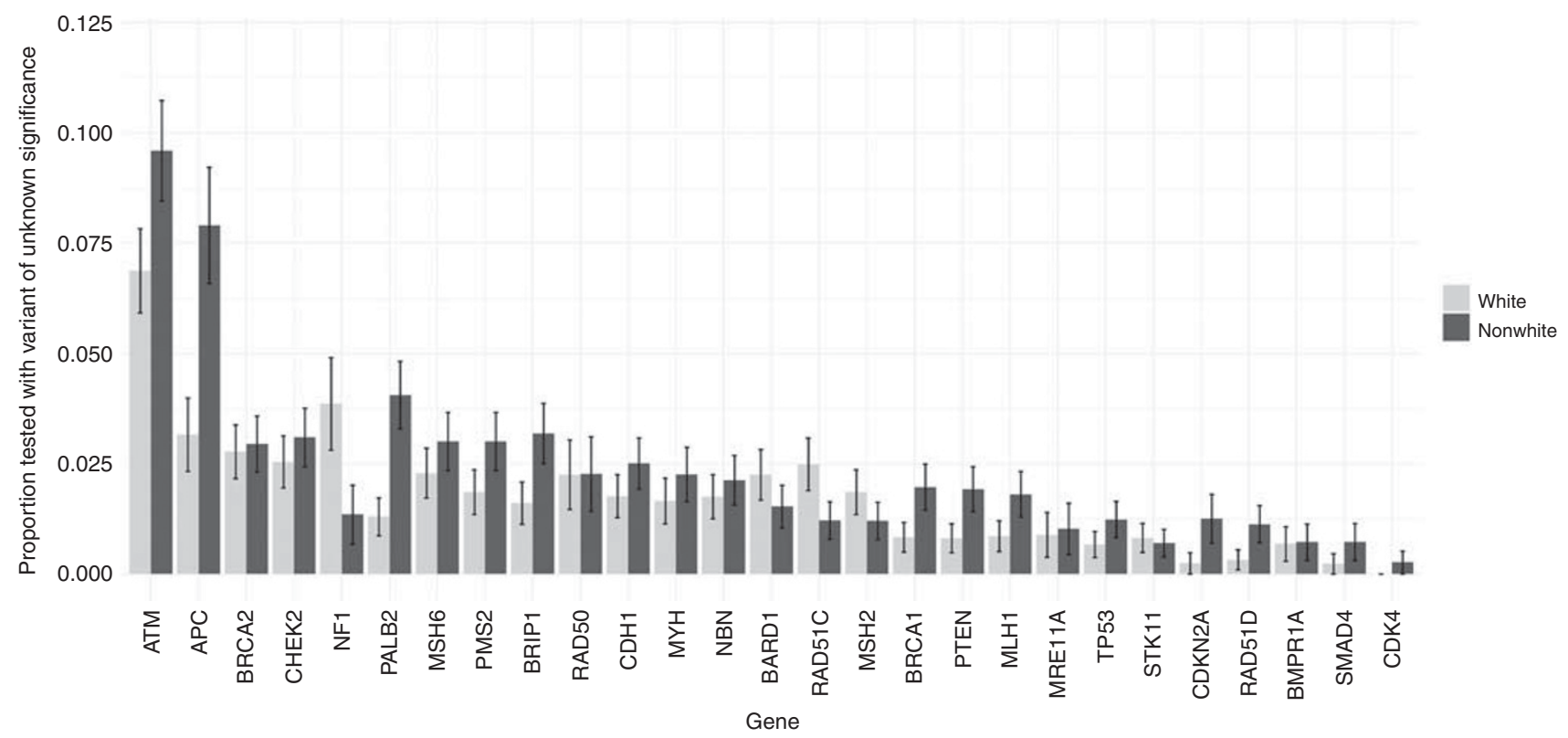

Figure 2 Rates of variants of unknown significance, by gene, across all patients tested for that gene. Error bars show \pm 1 standard deviation.

$(27 \%)(P=2 \mathrm{E}-4)$. VUS were most common in Asians (42\%), followed by Hispanics (31\%), then Ashkenazi Jews (30\%).

We next examined the effect of increasing gene number on panel-testing results (Figure 1b). We treated each paneltested patient as if $\mathrm{s} / \mathrm{he}$ were tested for $B R C A 1 / 2$ only $(N=1,432$; 51 patients were not tested for these two genes and were excluded from this analysis), then added five other known high-risk breast cancer genes $(N=1,331)$, then six moderate-risk breast/ovarian cancer genes $(N=1,237)$, and then the Lynch genes $(N=1,219)$. With each addition of genes to the panel, the number of patients in the group decreased, as a subset of patients was not tested for all genes. Finally, we created a cohort of patients tested for 23 genes by randomly selecting 5 additional genes for each patient who was tested for all 18 genes of interest, plus at least 5 additional genes $(N=1,052)$, and a cohort of patients tested for 28 genes by randomly selecting 5 additional genes for each patient tested for all 18 genes of interest, plus at least 10 more genes $(N=351)$. Increasing the number of genes increased the likelihood of a pathogenic variant, from $5.4 \%$ when only $B R C A 1 / 2$ were tested to $12 \%$ when 13 genes were tested. Beyond this point, we saw minimal improvement in pathogenic variant detection: $13 \%$ with 18 genes, $13 \%$ with 23 genes, and $12 \%$ with 28 genes (the decrease from 23 to 28 genes related to sampling error, with a smaller number of patients tested for 28 genes). Increasing gene number also increased the VUS rate, from $3.9 \%$ with BRCA1/2 only to $21 \%$ with 13 , to $30 \%$ with $23-8$ genes. The relation of VUS rate to panel size was amplified in nonwhites versus whites (Figure 1b): when only $B R C A 1 / 2$ were tested, the VUS rate was $4.4 \%$ in nonwhites compared to $3.3 \%$ in whites, while when 13 genes were tested, it was $25 \%$ in nonwhites compared to $17 \%$ in whites, and when 28 genes were tested, it was $38 \%$ in nonwhites compared to $25 \%$ in whites. Nineteen genes had a VUS rate of at least 1\%, with highest rates in ATM (8.2\%) and $A P C$ (5.5\%) (Figure 2).

\section{Pathogenic results by race/ethnicity}

We uncovered 235 pathogenic or likely pathogenic variants in 26 genes (Supplementary Table S2). The most commonly mutated gene was BRCA1 $(N=41)$, followed by BRCA2 $(N=40)$, then CHEK2 $(N=34)$. We assessed for differences in rates of pathogenic variants between whites and nonwhites in each of the seven genes in which we detected at least 10 pathogenic variants (BRCA1, BRCA2, CHEK2, MYH, ATM, $A P C, P A L B 2)$ (seven tests). After adjustment for multiple hypothesis testing, a significantly higher percentage of whites than nonwhites had a pathogenic variant in CHEK2 (3.8\% vs. $1.0 \% ; P=0.002)$, and a significantly lower percentage of whites than nonwhites had a pathogenic variant in $A P C(0.2 \%$ vs. $2.7 \% ; P=0.007)$. The difference in $A P C$ variants was driven entirely by the I1307K founder mutation in Ashkenazi Jewish patients; there was only one non-I1307K mutation identified out of 12 APC variants, in an Asian patient. There was a trend toward a lower percentage of whites than nonwhites with a pathogenic variant in PALB2 (0.3\% vs. $1.5 \%$; $P=0.02)$. Proportions of pathogenic or likely pathogenic results by race/ethnicity for each of thirteen genes associated with breast or ovarian cancer are shown in Figure 3.

\section{DISCUSSION}

We examined the results of hereditary cancer panel testing in a diverse population of patients who presented to an academic clinical cancer genetics program over a 2-year period, beginning after a US Supreme Court decision resulted in cheaper and more accessible multiple-gene panel testing. 

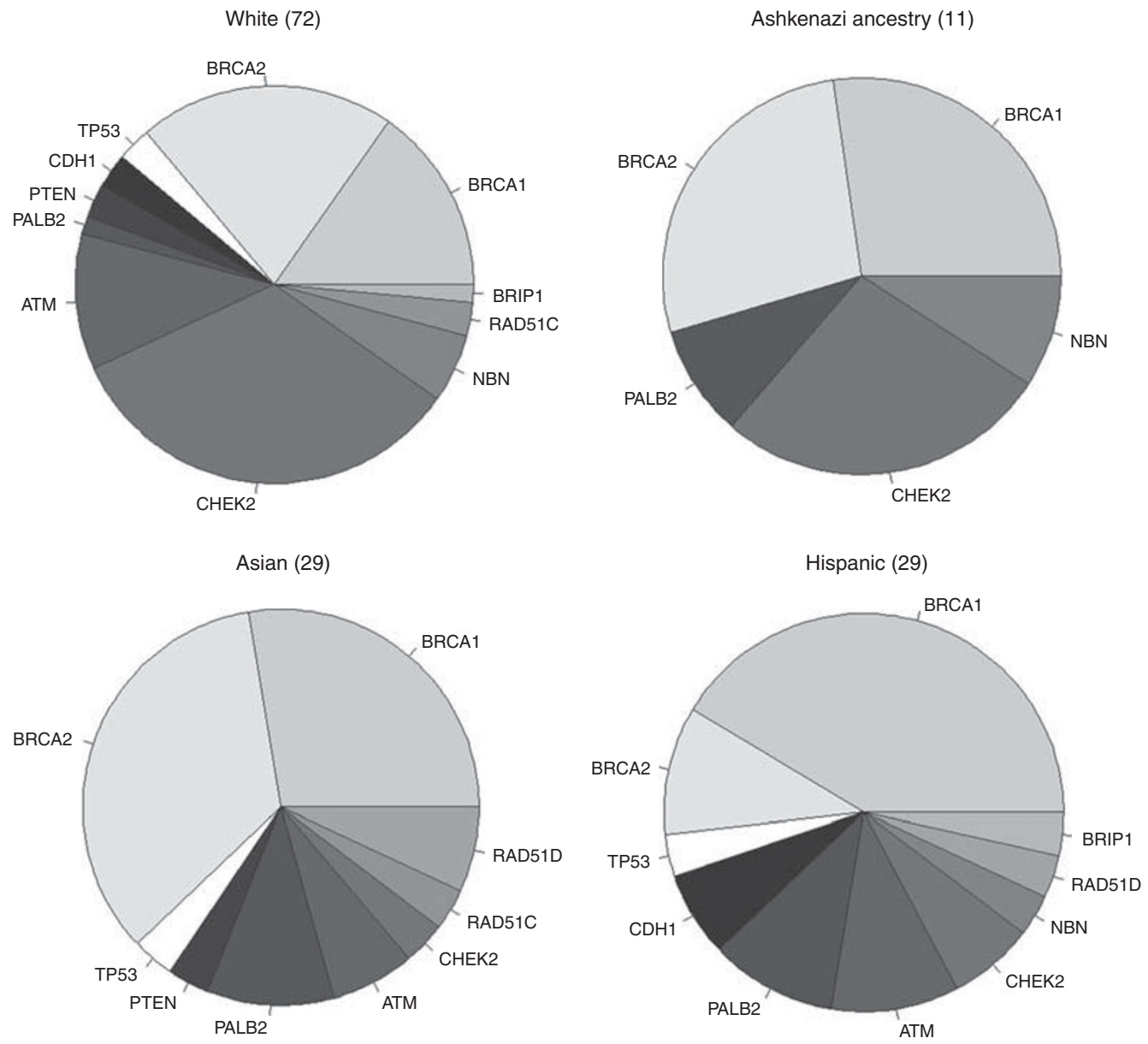

Figure 3 Pathogenic or likely pathogenic results by gene identified in the set of thirteen breast- or ovarian-cancer associated genes (BRCA1, BRCA2, TP53, CDH1, PTEN, STK11, PALB2, ATM, CHEK2, NBN, RAD51C, RAD51D, BRIP1). No pathogenic variants were found in STK11. The total number of pathogenic results identified in each racial/ethnic group is shown in parentheses.

We discovered important racial/ethnic differences in the demographics of patients presenting for testing and in their results. Compared to white patients, Asians and Hispanics were significantly younger and more likely to receive an uncertain result (VUS). Furthermore, the specific genes in which pathogenic variants were identified differed between races. Our results suggest racial discrepancies in patient referral for hereditary cancer panel testing and identify racial disparities in genetic results.

The proportion of VUS results (at least one VUS, with no pathogenic variant found) was significantly higher in nonwhites than in whites. Lower VUS rates among whites have been observed previously, ${ }^{8,9}$ and probably result from a greater proportion of whites than nonwhites in reference databases used to classify genetic variants. ${ }^{20}$ However, the implications of this finding are substantial. While most VUS are ultimately reclassified as benign, ${ }^{21}$ many clinicians report managing VUS results no differently from managing pathogenic variants. ${ }^{22}$ Thus, the greater incidence of VUS in nonwhites may propagate disparities in the quality of preventive care (for example, recommendations of unnecessary bilateral mastectomy or high-risk screening) and related harms. Furthermore, our discovery that the VUS disparity worsens with the number of genes tested is particularly relevant to the current clinical testing context, in which increasing numbers of genes are sequenced. This inequity must be remedied as precision medicine initiatives move forward.

We observed racial/ethnic differences among patients presenting for hereditary cancer panel testing. Asians and Hispanics were tested at younger ages than whites, while whites reported higher rates of family cancer history than Asians. One might speculate that clinicians elicited less complete family histories from Asian patients; it is also possible that Asian patients reported less family history, although we did not observe this in our previous work. ${ }^{23}$ There may also be a greater generational difference, where younger patients are more likely to pursue genetic testing than older patients, among Asians and Hispanics than among white patients. A higher proportion of Ashkenazi Jewish patients underwent MGS panel testing with no personal history of cancer, potentially reflecting a higher tendency to present to the clinic owing to ancestry alone, given their higher rates of $B R C A 1 / 2$ founder mutations.

The major strengths of our study are its racial/ethnic diversity and the real-world nature of referral indications and MGS 
panels ordered. Most previous work on clinical MGS has been limited to a single panel or commercial laboratory in predominantly white patients, ${ }^{3,5,9-11}$ limiting the generalizability of results. One limitation of the current study is its focus on only one academic cancer center and another is its relative dearth of African-American or Native American patients. Our results thus apply only to Asians and Hispanics, and specifically those Asian and Hispanic populations represented in the community: among Asian patients in our cohort, 83\% reported East Asian descent and 17\% reported South Asian descent, and while we did not collect information on subethnicity within Hispanic patients, approximately $80-90 \%$ of Californian and South Bay Hispanics are of Mexican descent. ${ }^{24,25}$ Notably, a previous study reported that VUS rates in patients of African descent were comparable to those in Asians. ${ }^{9}$ Similarly, another study focused on African Americans found a low proportion of pathogenic variants in $C H E K 2,{ }^{1}$ consistent with the lower rate of CHEK2 pathogenic variants in nonwhites that we report here.

In conclusion, we found important racial/ethnic differences in the characteristics of patients presenting for clinical panel testing; in the content of their panel testing results; and in the specific genes affected by pathogenic variants. Our findings should motivate particular urgency to reclassify VUS in nonwhites and thus mitigate the disparity in VUS rates that has followed a racial disparity in testing access. Our results also emphasize the importance of studying nonwhites to inform resource allocation for studies of pathogenic variants that are prevalent in specific subgroups (e.g., beyond the CHEK2 pathogenic variants that appear to predominate in whites). Further studies of MGS in diverse patient populations will be essential to close the widening racial gap in genetic panel testing results that threatens the successful implementation of emerging genetic technologies.

\section{SUPPLEMENTARY MATERIAL}

Supplementary material is linked to the online version of the paper at http://www.nature.com/gim

\section{ACKNOWLEDGMENTS}

This work was supported by the Suzanne Pride Bryan Fund for Breast Cancer Research, the Jan Weimer Junior Faculty Chair in Breast Oncology, the Breast Cancer Research Foundation, and the BRCA Foundation.

\section{DISCLOSURE}

J.M.F.'s work has been funded by Myriad Genetics and Natera. A. W.K.'s work has been funded by Myriad Genetics, Ambry Genetics, Invitae, and Genomic Health. The other authors declare no conflict of interest.

\section{REFERENCES}

1. Churpek JE, Walsh $T$, Zheng $Y$, et al. Inherited predisposition to breast cancer among African American women. Breast Cancer Res Treat. 2015;149:31-39.

2. Couch FJ, Hart SN, Sharma P, et al. Inherited mutations in 17 breast cancer susceptibility genes among a large triple-negative breast cancer cohort unselected for family history of breast cancer. I Clin Oncol 2015;33:304-311.

3. Desmond A, Kurian AW, Gabree $\mathrm{M}$, et al. Clinical actionability of multigene panel testing for hereditary breast and ovarian cancer risk assessment. JAMA Oncol. 2015;1:943-951.

4. Kurian AW, Hare EE, Mills MA, et al. Clinical evaluation of a multiplegene sequencing panel for hereditary cancer risk assessment. J Clin Oncol. 2014;32:2001-2009.

5. LaDuca H, Stuenkel AJ, Dolinsky JS, et al. Utilization of multigene panels in hereditary cancer predisposition testing: analysis of more than 2,000 patients. Genet Med 2014;16:830-837.

6. Maxwell KN, Wubbenhorst B, D'Andrea K, et al. Prevalence of mutations in a panel of breast cancer susceptibility genes in BRCA1/2-negative patients with early-onset breast cancer. Genet Med 2015;17:630-638.

7. Norquist BM, Harrell Ml, Brady MF, et al. Inherited mutations in women with ovarian carcinoma. JAMA Oncol. 2016;2:482-490.

8. Ricker C, Culver JO, Lowstuter K, et al. Increased yield of actionable mutations using multi-gene panels to assess hereditary cancer susceptibility in an ethnically diverse clinical cohort. Cancer Genet. 2016;209: 130-137.

9. Susswein LR, Marshall ML, Nusbaum R, et al. Pathogenic and likely pathogenic variant prevalence among the first 10,000 patients referred for next-generation cancer panel testing. Genet Med 2016;18: 823-832.

10. Thompson ER, Rowley SM, Li N, et al. Panel testing for familial breast cancer: calibrating the tension between research and clinical care. J Clin Oncol 2016;34:1455-1459.

11. Tung $N$, Battelli $C$, Allen $B$, et al. Frequency of mutations in individuals with breast cancer referred for BRCA1 and BRCA2 testing using next-generation sequencing with a 25-gene panel. Cancer 2015;121: 25-33.

12. Tung $\mathrm{N}$, Lin $\mathrm{NU}$, Kidd J, et al. Frequency of germline mutations in 25 cancer susceptibility genes in a sequential series of patients with breast cancer. J Clin Oncol 2016;34:1460-1468.

13. Daly MB, Pilarski R, Axilbund JE, et al. Genetic/familial high-risk assessment: breast and ovarian, Version 2.2015. J Natl Compr Canc Netw 2016;14:153-162.

14. Tung N, Domchek SM, Stadler Z, et al. Counselling framework for moderate-penetrance cancer-susceptibility mutations. Nat Rev Clin Oncol. 2016;13:581-588.

15. Manrai AK, Funke BH, Rehm HL, et al. Genetic misdiagnoses and the potential for health disparities. N Engl J Med 2016;375:655-665.

16. Spratt $D E$, Chan $T$, Waldron $L$, et al. Racial/ethnic disparities in genomic sequencing. JAMA Oncol. 2016;2:1070-1074.

17. Daly $M B$, Pilarski R, Axilbund JE, et al. Genetic/familial high-risk assessment: Breast and ovarian, version 2.2015. I Natl Compr Canc Netw 2016;14;153-162.

18. Provenzale D, Gupta S, Ahnen DJ, et al. Genetic/familial high-risk assessment: Colorectal version 1.2016. I Natl Compr Canc Netw 2016;14;1010-1030.

19. Kurian AW, Idos G, Culver J, et al. Safety of multiplex gene testing for inherited cancer risk: interim analysis of a clinical trial. Abstract presented at ASCO Annual Meeting. Chicago, IL, 3-7 June 2016.

20. Lek M, Karczewski KJ, Minikel EV, et al. Analysis of protein-coding genetic variation in 60,706 humans. Nature 2016;536:285-291.

21. Eggington JM, Bowles KR, Moyes K, et al. A comprehensive laboratorybased program for classification of variants of uncertain significance in hereditary cancer genes. Clin Genet 2014;86:229-237.

22. Kurian AW, Li Y, Hamilton AS, et al. Gaps in incorporating germline genetic testing into treatment decision-making for early-stage breast cancer. J Clin Oncol; e-pub ahead of print 12 April 2017.

23. Kurian AW, Gong GD, Chun NM, et al. Performance of BRCA1/2 mutation prediction models in Asian Americans. J Clin Oncol 2008;26: 4752-4758.

24. Brown A \& Lopez MH. Mapping the Latino population, by state, county and city. Pew Research Center's Hispanic Trend Projects, 2013. http:// www.pewhispanic.org/files/2013/08/latino_populations_in_the_states_ counties_and_cities_FINAL.pdf. Accessed 18 April 2017.

25. Pew Research Center. Hispanic population and origin in select U.S. metropolitan areas, 2014. http://uww.pewhispanic.org/interactives/ hispanic-population-in-select-u-s-metropolitan-areas/. Accessed 18 April 2017. 